\title{
The Effect of Using Oral Glutamine on the Side Effect of Mucositis in Patients with Head and Neck Cancer Who Are Receiving Chemoradiotherapy: Retrospective Evaluation with Clinical and Immunological Parameters
}

\author{
(1) Müge AKMANSU,' (1) Sevilay IREN, ${ }^{2}$ (1) Gül GÜNTÜRKÜN³ \\ 'Department of Radiation Oncology, Gazi University, School of Medicine, Ankara-Turkey \\ ${ }^{2}$ Department of Dr. Suat Seren Chest Diseases and Thoracic Surgery Training and Research Hospital, İzmir-Turkey \\ ${ }^{3}$ Department of Radiation Oncology, Meram Medical Faculty, Konya-Turkey
}

\begin{abstract}
OBJECTIVE
This study aimed to investigate the effect of glutamine supplementation on the intensity and duration of oral mucositis related to therapy and on proinflammatory cytokines in patients with head and neck cancer (HNC) who were treated with chemoradiotherapy (CRT).

\section{METHODS}

In total, 28 outpatients with HNC ( 24 male and 4 female) who were admitted to the Gazi University, Department of Radiation Oncology, between October 2008 and November 2009 were retrospectively evaluated. The mean age of the patients was 18-76 years. All the patients were histopathologically diagnosed with squamous cell carcinoma. Of the 28 patients who were included in the radiotherapy (RT) program, five were postoperatively included and 23 were included according to organ protection protocols. Eighteen patients prophylactically received oral powdered L-glutamine (Glutamine ResourceR, Nestle) starting from the first day of RT at a dose of $30 \mathrm{~g} / \mathrm{day}$ ( $10 \mathrm{~g}$ each at 8 -h intervals by mixing with water or fruit juice). For these patients, glutamine supplementation was continued until the completion of RT. Ten patients did not receive any supplementation product. At the initiation of RT, height and weight were measured for all the patients. The grade of oral mucositis was evaluated using the "Radiation Therapy Oncology Group (RTOG)" scoring system. During RT, changes in the weight of patients were routinely evaluated, whereas oral mucositis scoring was performed every week. At the beginning, during, and at the end of the therapy, serum IL-1 beta, IL-6, and TNF-alpha levels were measured, in addition to performing routine blood tests.
\end{abstract}

\section{RESULTS}

The incidences of grade 2 mucositis were comparable between the groups who received and did not receive glutamine supplementation ( $42.1 \%$ and $44.4 \%$, respectively). However, the incidence of severe mucositis (RTOG of $\geq$ grade 3 ) was significantly lower in the group receiving glutamine supplementation than in the group which did not receive glutamine supplementation $(5.3 \%$ and $55.6 \%$, respectively; $\mathrm{p}=0.008$ ). The day of onset of mucositis was the $18^{\text {th }}$ day of the therapy in the group that received glutamine supplementation and 14th in the group that did not receive glutamine supplementation, and this was statistically significant $(\mathrm{p}=0.007)$. Similarly, the median dose at which mucositis was started was $36 \mathrm{~Gy}$ in the group that received glutamine supplementation and $28 \mathrm{~Gy}$ in the group that did not receive glutamine supplementation $(\mathrm{p}=0.006)$. The two groups did not show any statistically significant difference in terms of weight change $(\mathrm{kg})$, percentage of weight change, and BMI $\left(\mathrm{kg} / \mathrm{m}^{2}\right)$ when the changes of weight were examined at the beginning and end of the treatment $(\mathrm{p}=0.42, \mathrm{p}=0.47$, and $\mathrm{p}=0.44$, respectively). When the changes in serum TNF-alpha, IL-1 beta, and IL-6 levels were compared at the beginning and end of the treatment, the groups did not show any significant difference $(\mathrm{p}=0.16, \mathrm{p}=0.08$, and $\mathrm{p}=0.77$, respectively).

\section{CONCLUSION}

Glutamine is a well-tolerated supplementation product with proven effects on oral mucositis, which is the side effect occurring during the therapy of HNCs. The effect of glutamine on inflammation leading to mucositis development in patients with HNC should be investigated in larger patient populations.

Keywords: Chemoradiotherapy; glutamine; irradiation; mucositis.

Copyright $\odot$ 2018, Turkish Society for Radiation Oncology 


\section{Introduction}

Head and neck cancers (HNCs) are the fifth most common cancers in the world. More than $50 \%$ of the cases are diagnosed during the locally advanced disease stage. Chemoradiotherapy (CRT), which is administered as a primary or postoperative adjuvant approach in the treatment of $\mathrm{HNC}$, has become a standard therapy since 10 years due to its successful results in providing a locoregional control and preventing metastasis. For the therapy, one or more chemotherapeutic agents may be used concomitantly with radiotherapy (RT). The most commonly used agents are cisplatin, 5-fluorouracil (5-FU), mitomycin $\mathrm{C}$, and taxane.[1,2,3] Oral mucositis is the most important side effect occurring during the treatment, which leads to an interruption in the treatment of patients by impairing the oral intake and causing malnutrition. The interruption of the therapy due to oral mucositis results in prolongation of the total duration of the therapy, which eventually leads to tumor repopulation and a decrease in the rate of locoregional control.[4] Due to RT, a local inflammation and an acute-phase response occur because of cell damage in the irradiated volume. This condition is caused by an increase in the levels of proinflammatory cytokines [tumor necrosis factor (TNF)-alpha and interleukin (IL)-6]. Local inflammation and irritation form the basis for the development of oral mucositis in patients with HNC.[5] Glutamine is a nonessential free amino acid that can be synthesized in the body. It is the precursor of nucleotides in DNA and RNA synthesis and plays a key role in lymphocyte proliferation. Glutathione (GSH/glutamyl-cysteinyl-glycine), which is the byproduct of GLN metabolism, is one of the antioxidants that have the highest concentrations in the cell and protects the normal tissue against oxidative damage.[6]

The level of GSH in tumor cells is 5-50 times higher than that in nontumor cells, and this high level of GSH mediates the resistance to CT and RT. $[7,8,9,10]$ The resistance for many anticancer agents is generally associated with increased levels of GSH in the tumor [11,12]; therefore, it is thought that reducing the levels of GSH in the tumor will increase the sensitivity of the tumor for RT and CT. In many studies, it has been shown that oral intake of GLN paradoxically reduces the levels of GSH in the tumor and increases them in the normal tissue. $[13,14,15,16]$ This is very important because GSH, which is the key regulator of cellular redox, determines the response of the tumor to CT and RT (simultaneously protects the normal cells). $[10,17,18]$ GLN supplementation reduces the levels of intratumoral
GSH and thereby prevents the continuation of tumor response to cytotoxic therapy.[19,20,21,22] This information suggests that GLN protects the normal tissue by increasing the selectivity of the antitumor therapy for the tumor and results in chemotherapeutical damage in the tumor cells by sensitizing them. As a result, a clear increase is seen in the therapeutic index. Few randomized studies have investigated the role of glutamine supplementation in the prevention of oral mucositis due to RT or CRT. These studies revealed that the prophylactic intravenous or oral use of glutamine decreased the intensity and duration of oral mucositis. $[3,4]$ In cell cultures, glutamine affected the production of cytokines such as TNF-alpha and IL-6, which are released from macrophages and neutrophils. $[23,24]$

This study aimed to investigate the effect of glutamine supplementation on the intensity and duration of oral mucositis related to therapy and on proinflammatory cytokines in patients with HNC who were treated with CRT.

\section{Materials and Methods}

A total of 28 outpatients with HNC (24 male and 4 female) who were admitted to the Gazi University Department of Radiation Oncology between October 2008 and November 2009 were retrospectively evaluated. The mean age of the patients was 18-76 years. Of the patients with an Eastern Cooperative Oncology Group (ECOG) performance score of $0-3$ at presentation to the clinic, 12 had nasopharyngeal, 11 had laryngeal, five had oropharyngeal, and one had hypopharyngeal carcinoma. At the time of diagnosis, all the patients had an ECOG performance score of $0-2$, except one patient with a locally advanced hypopharyngeal carcinoma who had a score of 3 . All the patients were histopathologically diagnosed with squamous cell carcinoma.

Of the 28 patients who were included in the RT program, five were postoperatively included and 23 were included according to organ protection protocols. While two patients received RT alone postoperatively, 26 (92\%) received chemotherapy (CT) regimens concomitantly with RT, and 6 (20\%) had received induction CT before concomitant $\mathrm{CT}$. Of the 26 patients who received CT, 13 received cisplatin, 11 received cisplatin and docetaxel, one received cisplatin, docetaxel, and 5-FU, and one received carboplatin concomitantly with RT.

\subsection{Radiotherapy}

After the immobilization of all patients using a thermoplastic fixation mask, their shoulders were pulled out 
using a string to exclude them from the therapy. After positioning the patient, treatment planning images were obtained using computed tomography (CT). The data obtained were transferred to the radiotherapy therapy planning system called Eclipse 7.2.02 (Varian Medical Systems, Canada, USA) in our clinic and were combined to obtain a 3D image. All targeted volumes, such as gross tumor volume, clinical targeted volume (CTV), and planned targeted volume, were drawn by a radiation oncologist according to ICRU-62 recommendations using axial CT images. TV was defined by considering the tolerated doses for critical organs, such as the spinal cord and mandible. RT was administered at doses and to sites which are routinely used in the clinical practice: conventional doses of 2 GY daily for every week and as five fractions per week. While one patient with a postoperative $\mathrm{RT}$ indication received 50 Gy to complete the therapy, four had an increase of the dose to 60-64 Gy at the primary tumor site by boosting after the administration of $50 \mathrm{~Gy}$. All the patients who were included in the organ protection protocol received a total of $70 \mathrm{~Gy}$ at the primary site with a boost after the administration of $50 \mathrm{~Gy}$.

\subsection{Administration of glutamine}

Eighteen patients prophylactically received oral powdered L-glutamine (Glutamine ResourceR, Nestle) starting from the first day of RT at a total dose of 30 $\mathrm{g} /$ day $(10 \mathrm{~g}$ at each $8 \mathrm{~h}$, by mixing with water or fruit juice). For these patients, glutamine supplementation was continued until the completion of RT. Ten patients did not receive any supplementation product.

\subsection{Evaluation of patients and oral mucositis scoring}

At the initiation of RT, height and weight were measured for all the patients. The grade of oral mucositis was evaluated using the Radiation Therapy Oncology Group (RTOG) scoring system. During RT, the changes in the weight of the patients were routinely evaluated, and oral mucositis scoring was performed every week. At the beginning, during, and at the end of the therapy, serum IL-1 beta, IL-6, and TNF-alpha levels were measured, in addition to performing routine blood tests.

\subsection{Statistical analysis}

For statistical analysis, patients were stratified according to their demographic characteristics and use of glutamine (yes/no). For the stratification, parameters such as age, sex, ECOG performance status, induction CT, and stage of the disease were used. While the distribution of frequency was used to describe the categorical variables, mean, median, and range were used for quantitative variables. Frequency distributions were compared using the Mann-Whitney, Chi-square, and Student's t test. A p-value of $<0.05$ was considered significant.

\section{Results}

In this retrospective study, 28 eligible patients were identified, and all the patients were included in the statistical analysis. None of the patients showed glutamine intolerance or glutamine-related toxicity. Table 1 shows the comparison between the therapy groups determined according to the use of glutamine (yes/no),

\begin{tabular}{|c|c|c|c|c|c|c|c|}
\hline \multirow[t]{2}{*}{ Parameters } & \multicolumn{2}{|c|}{ All patients } & \multicolumn{2}{|c|}{ Glutamine (+) } & \multicolumn{2}{|c|}{ Glutamine (-) } & \multirow[t]{2}{*}{$\mathbf{p}$} \\
\hline & $\mathbf{N}$ & (\%) & $\mathbf{N}$ & $(\%)$ & $\mathbf{N}$ & (\%) & \\
\hline \multicolumn{8}{|l|}{ Gender } \\
\hline Female & 4 & $(14.3)$ & 1 & (3.6) & 3 & (10.7) & 0.11 \\
\hline Male & 24 & (85.7) & 17 & $(60.7)$ & 7 & $(25)$ & \\
\hline \multicolumn{8}{|l|}{ Age } \\
\hline Median & 54 & 57 & 49 & 0.35 & & & \\
\hline Range & $(42-66)$ & $(48-66)$ & $(33-65)$ & & & & \\
\hline \multicolumn{8}{|l|}{ ECOG } \\
\hline $0-1$ & 22 & (78.5) & 15 & $(53.5)$ & 7 & $(25)$ & 0.35 \\
\hline 2 & 6 & (21.4) & 3 & (10.7) & 3 & (10.7) & \\
\hline \multicolumn{8}{|l|}{ Stage } \\
\hline $3 \mathrm{~A}$ & 2 & $(7,1)$ & 1 & (3.6) & 1 & (3.6) & 0.59 \\
\hline 3B & 26 & (21.4) & 17 & $(14.2)$ & 9 & (32.1) & \\
\hline \multicolumn{8}{|l|}{ Induction CT } \\
\hline Yes & 6 & (21.4) & 4 & $(14.2)$ & 2 & (7.1) & 0.64 \\
\hline No & 22 & (78.5) & 14 & $(50)$ & 8 & (28.5) & \\
\hline
\end{tabular}


Table 2 Therapeutic results

\begin{tabular}{|c|c|c|c|c|c|}
\hline \multirow{2}{*}{$\begin{array}{l}\text { Parameter } \\
\text { Oral mucositis grade }\end{array}$} & \multicolumn{2}{|c|}{ Glutamine (+) } & \multicolumn{2}{|c|}{ Glutamine (-) } & \multirow[t]{2}{*}{$\mathbf{p}$} \\
\hline & (N) & $(\%)$ & (N) & (\%) & \\
\hline $0-1$ & 9 & (32.1) & 0 & (0) & $0.003^{*}$ \\
\hline 2 & 8 & (28.5) & 3 & (10.7) & \\
\hline 3 & 1 & (3.57) & 7 & (21.4) & \\
\hline The day of onset of mucositis & 18 & & 14 & & $0.007^{*}$ \\
\hline \multicolumn{6}{|l|}{ Median } \\
\hline The dose of onset of mucositis/Gy & 36 & & 28 & & $0.006^{*}$ \\
\hline \multicolumn{6}{|l|}{ Median } \\
\hline Weight change (kg) & -6.1 & & -7.2 & & 0.42 \\
\hline \multicolumn{6}{|l|}{ Median } \\
\hline Percentage of weight change (\%) & -7.9 & & -9.4 & & 0.47 \\
\hline \multicolumn{6}{|l|}{ Median } \\
\hline BMI change $\left(\mathrm{kg} / \mathrm{m}^{2}\right)$ & 2.1 & & 2.5 & & 0.44 \\
\hline Median & & & & & \\
\hline
\end{tabular}

BMI: Body mass index

*Statistically significant.

by the parameters formed before the therapy. No statistically significant differences were found between the two groups. Table 2 shows the results of the therapy.

\subsection{Oral mucositis}

The incidences of grade 2 mucositis were comparable between the groups who received and did not receive glutamine supplementation $(42.1 \%$ and $44.4 \%$, respectively). However, the incidence of severe mucositis (RTOG of $\geq$ grade 3 ) was significantly lower in the group receiving glutamine supplementation than in the group not receiving it (5.3\% and $55.6 \%$, respectively; $\mathrm{p}=0.008$ ). In the group which did not receive glutamine supplementation, two patients were hospitalized due to severe mucositis. In the group which received oral glutamine, grade 1 toxicity was most commonly observed, with a rate of $47.4 \%$. The day of onset of mucositis was the $18^{\text {th }}$ day of the therapy in the group that received glutamine supplementation and 14th in the group that did not receive glutamine supplementation; this was statistically significant $(p=0.007)$. Similarly, the median dose at which mucositis was started was 36 Gy in the group that received glutamine supplementation and 28 Gy in the group that did not receive glutamine supplementation $(\mathrm{p}=0.006)$.

\subsection{Weight loss and BMI change}

The two therapy groups did not show any statistically significant difference in terms of weight change $(\mathrm{kg})$, percentage of weight change, and BMI $\left(\mathrm{kg} / \mathrm{m}^{2}\right)$ when the changes in weight were examined at the beginning and end of the treatment $(\mathrm{p}=0.42, \mathrm{p}=0.47$, and $\mathrm{p}=0.44$, respectively).

\subsection{Cytokine levels}

When the changes in serum TNF-alpha, IL-1 beta, and IL-6 levels were compared at the beginning and end of the treatment, the groups that received and did not receive glutamine supplementation did not show any significant difference $(\mathrm{p}=0.16, \mathrm{p}=0.08$, and $\mathrm{p}=0.77$, respectively).

\section{Discussion}

In patients with $\mathrm{HNC}$ who were treated with CRT, glutamine supplementation delayed the clinical onset of oral mucositis due to the therapy and reduced the intensity of mucositis. However, when weight changes of all the patients were evaluated at the beginning and end of the therapy, the two groups did not show any significant difference in terms of weight loss.

In patients with $\mathrm{HNC}$, the therapy includes RT, surgery, and CT, if required. Local inflammation and irritation related to RT results in side effects, such as mucositis, xerostomia, odinophagia, stomatitis, oesophagitis, and dysphagia.[25,26] Oral mucositis is the most important side effect in which the clinical symptoms are induced as a result of a loss in the epithelial cells. In patients who receive RT, mucositis generally appears within the second week of the therapy and continues until the end of the therapy with a gradually increasing intensity. In patients who receive $\mathrm{CT}$, it 
appears within 3-5 days after initiation of the therapy and peaks on days 7-10. Currently, there are no established methods to prevent or treat oral mucositis. For its treatment, many agents such as sucralphate, amiphostine, chlorhexidine, antimicrobial agents, and granulocyte-macrophage colony stimulating factor are being used, but the benefits of these agents in preventing or treating oral mucositis due to CRT are limited. $[27,28,29]$

Glutamine is a free amino acid that can be synthesized in the body. It is the precursor of both glutamate, which is required for the synthesis of glutathione and protects the cells from oxidative stress, and nucleotides playing a role in RNA and DNA synthesis. In their study, Klimberg et al. demonstrated the efficacy of glutamine, which is the main energy source of the intestinal epithelium, in the prevention of mucosal damage caused by radiation exposure in the intestine of rat models.[30,31,32] In addition, in the rats treated with methotrexate, the group that received glutamine supplementation showed a decrease in weight loss, increase in the mucosal weight of the jejunum and colon, longer lifespan, and lower incidence of bacteremia.[33] Many clinical studies have investigated the efficacy of glutamine in patients diagnosed with cancer during the last 20 years. Anderson et al. in their nonrandomized study which included 24 patients who received intensive CT regimens before bone marrow transplantation demonstrated that prophylactic use of oral glutamine reduces the intensity and duration of oral mucositis due to CT.[34] Recently, Peterson et al. in a randomized, placebo-controlled, multicenter, phase 3 study conducted on 326 patients with breast cancer receiving CT demonstrated that the clinical incidence and intensity of mucositis were significantly reduced in patients using glutamine compared with the placebo.[35]

When the Western style of nutrition is taken into consideration, dietary daily glutamine intake is generally $<10$ g. In humans, some catabolic conditions such as trauma and sepsis increase the need for glutamine, and quantity of daily dietary glutamine intake required to maintain homeostasis increases up to 20-40 g. [21] In patients with HNC, glutamine deficiency is an expected result. This leads to more severe manifestations of side effects that occur during the therapy. Because patients diagnosed with HNC have a history of smoking, use of alcohol, and malnutrition in general, deficiency of glutamine in addition to all these factors reduces the tolerance of the normal tissues to antineoplastic therapies. [36,37] The first study investigating the effect of glutamine on oral mucositis in patients receiving
RT or CRT for HNC was conducted by Huang et al., wherein 17 patients who received RT alone for HNC were randomized according to their use of prophylactic oral glutamine (yes/no). In this study conducted with a limited number of patients, it was found that the duration and intensity of oral mucositis significantly decreased in the group that received oral glutamine compared with the placebo group $(\mathrm{p} \leq 0.006)$.[4] In the double blind, placebo-controlled study of Cerchietti et al. that included 32 patients who received CRT for $\mathrm{HNC}$, patients were given L-alanine-L-glutamine combination via the intravenous route; the study found that the intensity of mucositis was significantly lower in the group receiving L-alanine-L-glutamine than in the placebo group ( $\mathrm{p}=0.007)$.[3] In this retrospective study, 28 patients who received primary or adjuvant CRT due to the diagnosis of HNC were evaluated. The patients who received oral glutamine during the therapy were compared with those who did not receive any nutritional supplementation. The patients who received glutamine supplementation were administered this product at a dose of $30 \mathrm{~g} /$ day as a prophylaxis starting from the beginning of the study. Parameters such as time of onset of mucositis due to the therapy, dose of radiotherapy at the time of first diagnosis, and severity of mucositis were investigated. The evaluation showed that glutamine supplementation in patients receiving CRT for HNC significantly delayed the onset and decreased the intensity of mucositis. In the group that did not receive glutamine supplementation, two patients were hospitalized due to severe mucositis after the interruption of their therapy. However, there were no patients who interrupted the therapy due to severe mucositis or other reason in the group who received the supplementation. The incidence of severe mucositis ( $\geq$ grade 3 ) was approximately $5 \%$ in the group that received the supplementation and approximately 55\% in the group that did not receive the supplementation $(\mathrm{p}=0.008)$. The two groups did not show any statistically significant difference in terms of weight loss due to malnutrition. This result was similar to the results obtained from the study of Huang et al. Thus, glutamine alone cannot prevent weight loss which gradually increases during the therapy. Therefore, an additional nutritional supplementation along with glutamine in patients with HNC may be efficient for the prevention of weight loss.

It is known that glutamine plays a key role in lymphocyte proliferation and affects the production of cytokines such as TNF-alpha and IL, which are released from macrophage and neutrophils. Pithon-Curi et al. demonstrated the effect of glutamine on cytokines 
levels in cell cultures.[23,24] Recently, Cruzat et al. found that plasma concentrations of TNF-alpha were lower in the rat groups who received free glutamine and L-alanine-L-glutamine dipeptide supplementation for the inflammatory response due to long term exercise than those in the control group $(\mathrm{p}<0.05)$. [38] Similarly, Fan et al. found that TNF-alpha were lower in burned rats who were given glutamine than in the controls $(p<0.05)$.[39] In their animal study, Ameho et al. investigated the effect of prophylactic glutamine on the occurrence of colitis, which develops in rats after exposure to trinitrobenzene sulphonic acid. In this study, although there was less damage and less bacterial translocation in the group which received GLN prophylaxis, the levels of IL-8 and TNF-alpha, which are strong inflammatory cytokines, was significantly lower than those in the control group. [40]

In HNC, locally administered RT leads to an inflammatory response in the irradiated volume. Due to cell damage, a local inflammation and an acutephase response are obserevd.[8,26] These conditions are caused by increased levels of proinflammatory cytokines (TNF-alpha and IL-6).[5,27] In our study, in all the patients, serum TNF-alpha, IL-1b, and IL-6 levels were measured at the beginning and end of the therapy, and responses for the following questions were elucidated: "In patients who receive RT for HNC, does glutamine decrease the inflammation due to RT? Is the positive effect of glutamine on oral mucositis correlated with the levels of these cytokines?" The serum levels of IL-6 measured at the end of the therapy were increased compared with those at the beginning of the therapy in both the groups. The levels of TNF-alpha and IL-1b at the end of the therapy were clinically reduced in the group that received glutamine supplementation compared with the group that did not; however, this was not statistically significant. This statistical result may be due to the limited number of patients evaluated and an unequal distribution of patients between the groups.

Based on this retrospective evaluation, we believe that there is a need for prospective, randomized studies including large patient samples to investigate the association between increased serum proinflammatory cytokine levels due to RT and prophylactic glutamine supplementation for reducing the intensity of mucositis in patients with HNC.

\section{Conclusion}

Glutamine is a well-tolerated supplementation product with proven effects on oral mucositis, which is the side effect occurring during the treatment of HNCs. The effect of glutamine on inflammation leading to mucositis development in patients with HNC should be investigated in larger patient populations.

Peer-review: Externally peer-reviewed.

Conflict of Interest: None declared.

\section{References}

1. Seiwert TY, Cohen EE. State-of-the-art management of locally advanced head and neck cancer. Br J Cancer 2005;92(8):1341-8.

2. Vermorken JB. Medical treatment in head and neck cancer. Ann Oncol 2005;16 Suppl 2:ii258-64.

3. Cerchietti LC, Navigante AH, Lutteral MA, Castro MA, Kirchuk R, Bonomi M, et al. Double-blinded, placebocontrolled trial on intravenous L-alanyl-L-glutamine in the incidence of oral mucositis following chemoradiotherapy in patients with head-and-neck cancer. Int J Radiat Oncol Biol Phys 2006;65(5):1330-7.

4. Huang EY, Leung SW, Wang CJ, Chen HC, Sun LM, Fang FM, et al. Oral glutamine to alleviate radiationinduced oral mucositis: a pilot randomized trial. Int J Radiat Oncol Biol Phys 2000;46(3):535-9.

5. Schlemmer M, Suchner U, Schäpers B, Duerr EM, Alteheld $\mathrm{B}$, Zwingers $\mathrm{T}$, et al. Is glutamine deficiency the link between inflammation, malnutrition, and fatigue in cancer patients? Clin Nutr 2015;34(6):1258-65.

6. Kosower NS, Kosower EM. The glutathione status of cells. Int Rev Cytol 1978;54:109-60.

7. Bozzetti F, Biganzoli L, Gavazzi C, Cappuzzo F, Carnaghi C, Buzzoni R, et al. Glutamine supplementation in cancer patients receiving chemotherapy: a double-blind randomized study. Nutrition 1997;13(7-8):748-51.

8. Brown SA, Goringe A, Fegan C, Davies SV, Giddings J, Whittaker JA, et al. Parenteral glutamine protects hepatic function during bone marrow transplantation. Bone Marrow Transplant 1998;22(3):281-4.

9. Bunpo P, Murray B, Cundiff J, Brizius E, Aldrich CJ, Anthony TG. Alanyl-glutamine consumption modifies the suppressive effect of L-asparaginase on lymphocyte populations in mice. J Nutr 2008;138(2):338-43.

10. Carretero J, Obrador E, Pellicer JA, Pascual A, Estrela JM. Mitochondrial glutathione depletion by glutamine in growing tumor cells. Free Radic Biol Med 2000;29(9):913-23.

11. Cao Y, Feng Z, Hoos A, Klimberg VS. Glutamine enhances gut glutathione production. JPEN J Parenter Enteral Nutr 1998;22(4):224-7.

12. Welbourne TC. Ammonia production and glutamine incorporation into glutathione in the functioning rat kidney. Can J Biochem 1979;57(3):233-7. 
13. Rouse K, Nwokedi E, Woodliff JE, Epstein J, Klimberg VS. Glutamine enhances selectivity of chemotherapy through changes in glutathione metabolism. Ann Surg 1995;221(4):420-6.

14. Arrick BA, Nathan CF. Glutathione metabolism as a determinant of therapeutic efficacy: a review. Cancer Res 1984;44(10):4224-32.

15. Wolf CR, Lewis AD, Carmichael J, Adams DJ, Allan SG, Ansell DJ. The role of glutathione in determining the response of normal and tumor cells to anticancer drugs. Biochem Soc Trans 1987;15(4):728-30.

16. Hong RW, Rounds JD, Helton WS, Robinson MK, Wilmore DW. Glutamine preserves liver glutathione after lethal hepatic injury. Ann Surg 1992;215(2):114-9.

17. Klimberg VS, Pappas AA, Nwokedi E, Jensen JC, Broadwater JR, Lang NP, et al. Effect of supplemental dietary glutamine on methotrexate concentrations in tumors. Arch Surg 1992;127(11):1317-20.

18. Klimberg VS, Nwokedi E, Hutchins LF, et al. Does glutamine facilitate chemotherapy while reducing its toxicity? Surg Forum 1991;42:16-18.

19. Wolf C, Hayward I, Lawrie S, et al. Cisplatinum-sensitive and resistant ovarian adenocarsinoma cell lines derived from the same patient(abstract). Proc Am Assoc Cancer Res 1985;26:338

20.Ziegler TR, Benfell K, Smith RJ, Young LS, Brown E, Ferrari-Baliviera E, et al. Safety and metabolic effects of L-glutamine administration in humans. JPEN J Parenter Enteral Nutr 1990;14(4 Suppl):137S-146S.

21. Windmueller HG. Glutamine utilization by the small intestine. Adv Enzymol Relat Areas Mol Biol 1982;53:201-37.

22. Panigrahi P, Gewolb IH, Bamford P, Horvath K. Role of glutamine in bacterial transcytosis and epithelial cell injury. JPEN J Parenter Enteral Nutr 1997;21(2):75-80.

23. Pithon-Curi TC, Trezena AG, Tavares-Lima W, Curi R. Evidence that glutamine is involved in neutrophil function. Cell Biochem Funct 2002;20(2):81-6.

24. Newsholme P, Procopio J, Lima MM, Pithon-Curi TC, Curi R. Glutamine and glutamate--their central role in cell metabolism and function. Cell Biochem Funct 2003;21(1):1-9.

25. Örnek çalıştay

26. Örnek çalıştay

27. Lievens Y, Haustermans K, Van den Weyngaert D, Van den Bogaert W, Scalliet P, Hutsebaut L, et al. Does sucralfate reduce the acute side-effects in head and neck cancer treated with radiotherapy? A double-blind randomized trial. Radiother Oncol 1998;47(2):149-53.

28. Martin MV, van Saene HK. The role of oral microorganisms in cancer therapy. Curr Opin Dent 1992;2:81-4.
29. Abitbol AA, Sridhar KS, Lewin AA, Schwade JG, Raub W Jr, Wolfson A, et al. Hyperfractionated radiation therapy and 5-fluorouracil, cisplatin, and mitomycinC (+/- granulocyte-colony stimulating factor) in the treatment of patients with locally advanced head and neck carcinoma. Cancer 1997;80(2):266-76.

30. Klimberg VS, Souba WW, Dolson DJ, Salloum RM, Hautamaki RD, Plumley DA, et al. Prophylactic glutamine protects the intestinal mucosa from radiation injury. Cancer 1990;66(1):62-8.

31. Souba WW, Klimberg VS, Copeland EM 3rd. Glutamine nutrition in the management of radiation enteritis. JPEN J Parenter Enteral Nutr 1990;14(4 Suppl):106S-108S.

32. Shou J, Lieberman MD, Hofmann K, Leon P, Redmond HP, Davies $\mathrm{H}$, et al. Dietary manipulation of methotrexate-induced enterocolitis. JPEN J Parenter Enteral Nutr 1991;15(3):307-12.

33. Fox AD, Kripke SA, De Paula J, Berman JM, Settle RG, Rombeau JL. Effect of a glutamine-supplemented enteral diet on methotrexate-induced enterocolitis. JPEN J Parenter Enteral Nutr 1988;12(4):325-31.

34. Anderson PM, Schroeder G, Skubitz KM. Oral glutamine reduces the duration and severity of stomatitis after cytotoxic cancerchemotherapy. Cancer 1998;83(7):1433-9.

35. Peterson DE, Jones JB, Petit RG 2nd. Randomized, placebo-controlled trial of Saforis for prevention and treatment of oral mucositis in breast cancer patients receiving anthracycline-based chemotherapy. Cancer 2007;109(2):322-31.

36. Savarese DM, Savy G, Vahdat L, Wischmeyer PE, Corey B. Prevention of chemotherapy and radiation toxicity with glutamine. Cancer Treat Rev 2003;29(6):501-13.

37. Noé JE. L-glutamine use in the treatment and prevention of mucositis and cachexia: a naturopathic perspective. Integr Cancer Ther 2009;8(4):409-15.

38. Cruzat VF, Rogero MM, Tirapegui J. Effects of supplementation with free glutamine and the dipeptide alanyl-glutamine on parameters of muscle damage and inflammation in rats submitted to prolonged exercise. Cell Biochem Funct 2010;28(1):24-30.

39. Fan J, Meng Q, Guo G, Xie Y, Li X, Xiu Y, et al. Effects of glutamine added to enteral nutrition on Peyer's patch apoptosis in severely burned mice. Burns 2010;36(3):409-17.

40. Ameho CK, Adjei AA, Harrison EK, Takeshita K, Morioka T, Arakaki Y, et al. Prophylactic effect of dietary glutamine supplementation on interleukin 8 and tumour necrosisfactor alpha production in trinitrobenzene sulphonic acid induced colitis. Gut 1997;41(4):487-93. 